\title{
Specific immunotherapy in hepatocellular cancer: A systematic review
}

\author{
Behnoud Baradaran Noveiry ${ }^{*}$, Armin Hirbod-Mobarakeh ${ }^{\star}, \dagger, \ddagger$, Nastaran Khalili ${ }^{*}$, Niloufar \\ Hourshad", Tim F Greten§, Ghassan K Abou-Alfa ${ }^{\star *}{ }^{* *}$, and Nima Rezaei ${ }^{*}, \dagger, \neq, \dagger \dagger$
}

*Border of Immune Tolerance Education and Research Network (BITERN), Universal Scientific Education and Research Network (USERN), Tehran, Iran ${ }^{\dagger}$ Molecular Immunology Research Center, Department of Immunology, School of Medicine, Tehran, Iran ₹Research Center for Immunodeficiencies, Children's Medical Center, Tehran University of Medical Sciences, Tehran, Iran §Gastrointestinal Malignancy Section, Thoracic and Gastrointestinal Oncology Branch, Center for Cancer Research, National Cancer Institute, NIH, Bethesda, Maryland ${ }^{\top}$ Memorial Sloan Kettering Cancer Center, New York, USA **Weill Cornell Medical College, New York, USA t†Systematic Review and Meta-analysis Expert Group (SRMEG), Universal Scientific Education and Research Network (USERN), Sheffield, UK

\section{Abstract}

Background and Aim: In recent years, several novel immunotherapeutic approaches were developed and investigated in patients with hepatocellular carcinoma (HCC). We designed this systematic review, to evaluate clinical efficacy of specific immunotherapy in patients with HCC, according to the guidelines of Border of Immune Tolerance Education and Research Network (BITERN) and Cochrane collaboration.

Methods: We searched Medline, Scopus, CENTRAL, TRIP, DART, OpenGrey, and ProQuest through the 9th of December 2015. One author reviewed and retrieved citations from these seven databases for irrelevant and duplicate studies, and two other authors independently extracted data from the studies and rated their quality. We collated study findings and calculated a weighted treatment effect across studies using Review Manager.

Results: We found 12144 references in seven databases of which 21 controlled studies with 1885 HCC patients in different stages were included in this systematic review after the primary and secondary screenings. Overall, patients undergoing specific immunotherapy had significantly higher overall survival than those in control group (HR $=0.59 ; 95 \% \mathrm{CI}=0.47-0.76, P<0.0001)$. There was a significant difference in recurrence-free survival between patients undergoing specific

Correspondence: Nima Rezaei, Research Center for Immunodeficiencies, Children's Medical Center, Hospital, Dr Qarib St, Keshavarz Blvd, Tehran, 14194, Iran. rezaei_nima@tums.ac.ir.

Conflict of interest statement: Hereby, we state that there is no ethical problem (approved by the research ethics committee of Tehran University of Medical Sciences) or conflict of interest in our research. There wasn't any honorarium, grant, or other form of payment to authors to produce the manuscript.

Supporting information

Additional Supporting Information may be found in the online version of this article at the publisher's web-site: Appendix S1. Systematic Review Protocol.

Appendix S2. Tool for assessing risk of bias in clinical trials. 
immunotherapy and patients in control groups and patients in immunotherapy groups overall had less recurrence than control group $(\mathrm{HR}=0.54 ; 95 \% \mathrm{CI}=0.46-0.63, P<0.00001)$.

Conclusions: Results of this systematic review based on the available literature suggest that overall specific immunotherapeutic approaches could be beneficiary for the treatment of patients with HCC. This further supports the current and ongoing evaluations of specific immunotherapies in the field.

\section{Keywords}

active immunotherapy; clinical outcomes; hepatocellular carcinoma; immunotherapy; metaanalysis; passive immunotherapy; systematic review; vaccines

\section{Introduction}

Hepatocellular carcinoma (HCC) is the fifth most common cancer and the third leading cause of cancer-related mortality worldwide. ${ }^{1}$ Multiple therapeutic modalities can be applied in patients with HCC depending on factors such as the degree of liver function, tumor burden, and associated comorbidities. Curative treatment options for early stage $\mathrm{HCC}$ are surgical resection or orthotopic liver transplantation (OLT). ${ }^{2}$ Other methods for early but non-resectable HCC include minimally invasive approaches such as percutaneous ethanol injection (PEA) and radiofrequency ablation (RFA). ${ }^{2,3}$ Trans-arterial chemoembolization (TACE) is currently considered as first line therapy in patients with intermediate-stage disease. ${ }^{3}$ However, most patients with HCC presents with advanced disease. ${ }^{2}$ Multi-drug resistance (MDR) genes are reported to be highly expressed in HCCs; therefore, HCC is extremely chemo-resistant. ${ }^{4}$ In addition, due to underlying cirrhosis and impaired drug metabolism in most patients with HCC, chemotherapy results in devastating adverse effects.

There are several reports of spontaneous regression in patients with HCC, for which antitumor immunity has been known to play a leading role. ${ }^{5,6}$ The observation of spontaneous regression of HCCs, as a consequence of the host immune response, paved the way toward development of novel passive and active immunotherapeutic approaches in the management of advanced HCC. ${ }^{5,6} \mathrm{HCC}$ is a typical inflammation induced cancer, which makes it possibly more susceptible to an immune based approach. Unlike, nonselective effects of conventional treatments, immunotherapy, theoretically, could selectively target and destroy malignant cells with minimal side effects. ${ }^{7-11}$ Passive immunotherapy involves administrating components of immune system such as immune cells or antibodies to patients to provide immunity against tumor cells. ${ }^{7-11}$ Onthe other hand, active immunotherapy stimulates host immune responses to recognize and destroy malignant cells. ${ }^{7-11}$

We designed this systematic review to evaluate clinical efficacy of specific immunotherapies in patients with HCC, according to the guidelines of Border of Immune Tolerance Education and Research Network (BITERN) and Cochrane collaboration. 


\section{Methods}

We conducted this systematic review in accordance with general methods recommended by Cochrane collaboration and Border of Immune Tolerance Education and Research Network guidelines.

\section{Inclusion criteria for considering studies for this review.}

In this systematic review, only controlled clinical trials (irrespective of blinding and randomization) investigating clinical efficacy of specific immunotherapies (irrespective of modes of administration, dosage, frequency and duration) in patients with primary HCC (irrespective of disease stage, age, gender or race) according to inclusion criteria stated in the protocol were included (Appendix S1). According to the protocol, phase 1 clinical trials without control or studies investigating non-specific immunotherapies such as immunomodulatory antibodies and immune checkpoints inhibitors and cytokine therapies were not included in this systematic review, considering the early nature of their development and their declaration of a new era that is currently being studied further.

\section{Literature search strategy for identification of studies.}

The primary search process was conducted in Medline, Scopus, TRIP, and CENTRAL databases (as databases for journal articles) and DART, OpenGrey, and ProQuest (as databases for grey literature) according to the search strategies described in the protocol (Appendix S1) on 18 May 2014. We updated the search process in Medline, Scopus, CENTRAL, ProQuest, DART, and OpenGrey databases up to the 9th of December 2015.

One of the authors imported citations from all databases into an Endnote library (version X6, Thomson Reuters, USA), removed duplicate citations using the "Find Duplicates" feature of Endnote software and then removed obviously irrelevant studies by screening title and abstract of the remainders of search results. We then retrieved full texts of remainder of citations for further screening and data collection process and quality assessment.

\section{Data extraction and quality assessment.}

Two of authors carefully read full texts of articles and independently included eligible articles based on the inclusion criteria stated in the protocol (Appendix S1). Disagreements between two authors were resolved by discussion or consultation with a third author and a final consensus.

Two authors independently extracted data including study title, corresponding author name, population information (sample size, age, and gender of participants), type of study, intervention, inclusion criteria, outcomes, and bias assessment from full texts of articles and entered them to pre-designed data extraction forms in Microsoft Excel spreadsheets (version 2010, Microsoft Corporation, USA).

Two reviewers independently evaluated studies for risk of bias in six domains of random sequence generation (selection bias for controlled trials), allocation concealment (selection bias for controlled trials), blinding of participants and personnel (performance bias), blinding of outcome assessment (detection bias), incomplete outcome data (attrition bias) 
and selective reporting (reporting bias) according to the "Risk of bias" tool (Appendix S2) and attributed each domain to be of "low risk" of bias, "high risk" of bias, or "unclear risk" of bias for each article. Any disagreement between two authors regarding quality of studies was resolved through discussion or consultation with the third author.

\section{Evidence synthesis.}

We did evidence synthesis using systematic approaches like tabulation and textual description, and we converted results from each study into a common rubric using Review Manager (Version 5.3. Copenhagen: The Nordic Cochrane Centre, the Cochrane Collaboration, 2014). For missing data, we used statistical methods to calculate missing data using data presented in the articles or in some cases we contacted the corresponding author.

We used Review Manager to do meta-analysis and calculate a weighted treatment effect across studies. We evaluated between-study heterogeneity by the chi-square statistic and its $\mathrm{P}$ value and $\mathrm{I}^{2}$ statistic. We considered a $P$ value $<0.1$ and $\mathrm{I}^{2}>40 \%$ as indicating significant between-study heterogeneity. In case of significant statistical heterogeneity or clinical heterogeneity, a random-effects model was used to do meta-analysis. In addition, we used sub-group analysis in case of clinical heterogeneity. We expressed the results of metaanalysis in hazard ratio (HR) with $95 \%$ confidence intervals (CI) for time-to-event outcomes and risk ratio (RR) with $95 \% \mathrm{CI}$ for dichotomous outcomes.

\section{Results}

\section{Description of included studies.}

We found 12144 references by applying search strategy in four databases of journal articles and three databases of grey literature. We then discarded 1847 duplicate citations using Endnote software and 10084 articles due to obvious irrelevancy of their topics in primary screening (Fig. 1). In secondary screening of 213 full texts, we excluded 192 articles, and finally included 21 controlled trials with $1885 \mathrm{HCC}$ patients in this systematic review (Table $1)$.

Overall, six studies with 254 patients addressed two active immunotherapeutic approaches including autologous formalin- fixed tumor vaccine (AFTV) ${ }^{12-15}$ and dendritic cell (DC)based vaccines. ${ }^{16-18}$ Twelve studies with 1459 patients investigated passive immunotherapeutic approaches including autologous cytokine-induced killer (CIK) cells, ${ }^{19-27}$ auto lymphocyte therapy (ALT), ${ }^{28}$ autologous lymphokine-activated killer (LAK) cells $7,26,29$ and radio immunotherapy (RIT). ${ }^{30-33}$ The clinical efficacy of pulsed DCs, an active immunotherapy, with CIK cells, a passive immunotherapy was studied in three controlled trials with 172 patients concurrently. ${ }^{34-36}$ All immunotherapeutic approaches reported in these studies were administered systemically.

Clinical outcomes investigated in these studies included overall survival, progression-free survival, recurrence-free survival, and toxicity.

The overall quality of studies was fair. There was high risk of selection bias in studies of Shimizu et al. and Hao et al. as they did not apply randomization methods to allocate 
patients to treatment arms (Table 2). ${ }^{25,34}$ In addition, the randomization method was not described in majority of studies and therefore, the risk of selection bias was unknown. Majority of studies did not use placebo or blinding methods to prevent performance bias. There was no risk of attrition bias for studies (Table 2).

\section{Overall survival.}

Overall survival and mortality were reported in 17 studies of which data on hazard ratio could have been retrieved from 15 studies.

Considering the presence of moderate statistical heterogeneity and clinical heterogeneity between results of studies $\left(\mathrm{Tau}^{2}=0.08 ; P=0.05 ; \mathrm{I}^{2}=40 \%\right.$ ), we employed subgroup analysis and random effects model to perform a meta-analysis on results of these 15 studies. Overall survival was significantly higher in patients who underwent specific immunotherapy comparing to patients in control groups $(\mathrm{HR}=0.59$; 95\% $\mathrm{CI}=0.47-0.76, P<0.0001)$ (Fig. 2).

Active immunotherapies.-Gao et al. and Ansary et al. reported that treatment with DCbased vaccines had a significant beneficiary effect on overall survival of HCC patients (Table 1). ${ }^{16,17}$ However, Nakamoto et al. reported that vaccination with immature DCs did not have any beneficial effect on overall survival (Table 1$).{ }^{18}$

Kuang et al. reported that in subgroup analysis for tumor size in 39 patients, AFTV therapy significantly improved overall survival in patients with tumor size $\geq 50 \mathrm{~mm}{ }^{12,13}$

Passive immunotherapy.-In subgroup meta-analysis of studies on passive specific immunotherapy, patients undergoing passive immunotherapy had significantly higher overall survival than those in control group ( $\mathrm{HR}=0.6 ; 95 \% \mathrm{CI}=0.44-0.8, P=0.0006)$. In subgroup meta-analysis of five studies on CIK therapy, CIK cell transfusion significantly prolonged overall survival $(\mathrm{HR}=0.5 ; 95 \% \mathrm{CI}=0.35-0.71, P=0.0001)$.

Combined immunotherapies.-In subgroup meta-analysis of studies investigating combined immunotherapeutic approaches (autologous DC-CIK cell therapy), patients in experimental groups had higher overall survival than those in control groups although this difference was not significant $(\mathrm{HR}=0.58 ; 95 \% \mathrm{CI}=0.31-1.09, P=0.09)$.

\section{Recurrence-free survival and progression-free survival.}

Recurrence-free survival was reported in 13 studies including four studies on active immunotherapy and eight studies on passive immunotherapy and one study on combined immunotherapy with 94 patients reported data on hazard ratio of recurrence between experimental and control groups.

Although there was not any statistical heterogeneity between results of these 13 studies, considering the presence of clinical heterogeneity, we employed subgroup analysis and random-effects model to conduct meta-analysis. Overall, patients undergoing specific immunotherapy had significantly higher recurrence-free survival than patients in control groups (HR $=0.54 ; 95 \% \mathrm{CI}=0.46-0.63, P<0.00001)$ (Fig. 3). 
Active immunotherapies.-In subgroup meta-analysis of four studies on active immunotherapy with 194 patients, patients undergoing active immunotherapy had significantly higher recurrence free survival $(\mathrm{HR}=0.59 ; 95 \% \mathrm{CI}=0.40-0.85, P=0.005)$. Although, three studies on AFTV did not report any significant difference in recurrence rate between patients in AFTV and control arms, in pooled analysis, AFTV significantly improved recurrence free survival in patients with $\mathrm{HCC}(\mathrm{HR}=0.55 ; 95 \% \mathrm{CI}=0.35-0.87, P$ $=0.01$ ). In addition, Gao et al. reported a significant lower recurrence rate after 1.5 years in patients undergoing DC-based vaccination than controls (Table 1). ${ }^{16}$

Kuang et al. reported that in subgroup analysis for tumor size in 39 patients, AFTV therapy significantly improved recurrence-free survival in patients with tumor size $250 \mathrm{~mm}{ }^{12,13}$

Passive immunotherapies.-In subgroup meta-analysis of eight studies on passive immunotherapy with 1018 patients, recurrence-free survival was significantly higher in patients undergoing passive immunotherapy $(\mathrm{HR}=0.5 ; 95 \% \mathrm{CI}=0.40-0.62, P<0.00001)$. In subgroup meta-analysis of three studies investigating CIK therapy, hazard ratio of recurrence was significantly lower in patients in experimental groups $(\mathrm{HR}=0.41 ; 95 \% \mathrm{CI}=$ $0.23-0.76, P=0.004) .{ }^{19,20,27}$ In addition, Hao et al. and Yu et al. both reported beneficial effect of CIK cell therapy in patients with HCC on progression free survival (Table 1). ${ }^{25,26}$

Lee et al. reported that in subgroup analysis for etiology of HCC in 186 patients, CIK cell therapy significantly improved recurrence-free survival in patients with HBV but this improvement in recurrence-free survival was not statistically significant in patients with $\mathrm{HCV}$ or other etiologies. ${ }^{19}$ In subgroup analysis for a number of Clinicopathophysiologic characteristics, Bian et al. reported that Licartin therapy significantly improved recurrencefree survival in patients with BCLC stage 0-A $(n=94)$, tumor size $30 \mathrm{~mm}(n=49)$ and single lesion $(n=66)^{30}$

Combined immunotherapies.-Shimuzu et al. reported that patients in combined specific immunotherapy arm had higher recurrence-free survival than those in control arm however this difference was not significant. ${ }^{34}$

\section{Safety.}

Overall, 20 studies reported safety parameters in patients undergoing specific immunotherapy. Overall, specific immunotherapies were safe and comparing to standard treatments resulted in even less serious adverse events although minor shortterm side effects as fever (mostly), allergic reactions, headache and pain were reported (Table 1).

Discussion.-We used a comprehensive search strategy in seven databases to retrieve controlled clinical trials investigating specific immunotherapy and presented and analyzed results of 21 retrieved articles with $1885 \mathrm{HCC}$ patients in a comprehensive and systematic manner. The retrieved studies were heterogeneous in regard of the immunotherapeutic approaches they reported and also their study populations. Therefore, we collected and presented details of each study in our systematic review. In addition, we used random effects and sub-group meta-analysis based on different approaches of immunotherapy used in these studies. 


\section{Active immunotherapy.}

Overall, two active immunotherapeutic approaches including AFTV and DC-based vaccination were reported in 6 clinical trials.

This systematic review showed positive effect of AFTV, an autologous tumor vaccine fixed with formalin, on recurrence-free survival. The patient's immune system can recognize tumor antigens present in autologous fixed tumor lysates and therefore can elicit a specific antitumor response. ${ }^{12,14,15}$ In addition, sustained release of GM-CSF and IL-2 from microparticles, administered along with AFTV, persistently activates antigen presenting cells (APCs) and cytotoxic T lymphocytes (CTLs) and induces longterm stimulation of the immune system and persistent antitumor immunity. ${ }^{12,14}$

On the other hand, AFTV contains some normal tissues in addition to malignant cells. Antigens from these normal liver cells can be uptaken by APCs and result in an autoimmune response against normal cells of liver. ${ }^{14}$ However, none of studies reported an autoimmune disease in patients under treatment with AFTV. ${ }^{12,14}$

Our systematic review showed that vaccination with antigen-preloaded DCs, one of the novel modalities of immunotherapy in cancer, had a beneficiary effect on overall survival and recurrence-free survival. ${ }^{16,17}$ DCs, as the most potent APCs, express high levels of the major histocompatibility complex (MHCs) and various costimulatory molecules and cytokines and therefore can effectively absorb and process tumor antigens to effector immune cells and elicit strong anti-tumor immune response. ${ }^{8,9,16,17,37}$ However, infusion of immature DCs did not improve overall survival or recurrence-free survival which might be due to insufficient antigens to be taken up by DCs after the infusion. ${ }^{7,10,18,37}$

Although DC vaccination was effective in terms of overall survival and recurrence free survival, these approaches are too cumbersome and costly. ${ }^{12,15}$

\section{Passive immunotherapy.}

Overall, four passive immunotherapeutic approaches including autologous CIK cell therapy, ${ }^{19-27}$ ALT, ${ }^{28}$ autologous LAK cell therapy ${ }^{7,26,29}$ and RIT $^{30-33}$ were reported in twelve clinical trials.

Our systematic review showed that CIK therapy is effective in terms of prolonging overall survival, recurrence-free survival and progression-free survival. CIK cells are non MHCrestricted cytotoxic lymphocytes with the characteristic $\mathrm{CD}^{+}-\mathrm{CD} 6^{+}$phenotype. ${ }^{38,39}$ They can be generated by addition of various cytokines including IL-2, IL-1, and IFN- $\gamma$ to peripheral blood monocytes. ${ }^{8,20,38}$ CIK cells can traffic efficiently to tumor sites and produce a strong anti-tumor response. ${ }^{21,25}$ In addition, they can shift the immune response toward a TH1 direction by producing effector cytokines (such as IL2, IL6, and IFN $\gamma$ ) and chemokines. ${ }^{8-10,21,25}$ Lee et al. also found significant improvement in recurrence-free survival in patients with Hepatitis B but not in group of patients with hepatitis $\mathrm{C}$, although lack of significant effect in this group might be due to few numbers of patients in this subgroup. However, CIK cells might reduce tumor recurrence by suppressing the replication of HBV. ${ }^{19}$ 
Results from this systematic review revealed that LAK cells are effective in prolonging recurrence free survival in patients with HCC. LAK cells consist of a diverse population of cells including primarily cytotoxic natural killer cells and T cells. ${ }^{7,26,29,40,41}$

In ALT, lymphokine activated autologous lymphocytes depleted from suppressor cells are transfused back to the patients. ${ }^{28,39,42}$

RIT is one of the more attractive approaches to cancer treatment in which radioactive tumoricidal agents are delivered to tumor cells using radio-conjugated monoclonal antibodies (mAbs) against tumor antigens. ${ }^{7-10,31}$ Licartin ( ${ }^{131}$ I-metuximab injection), one of novel radio-conjugated $\mathrm{mAbs}$, is produced by labeling metuximab with iodine-131..$^{30,33}$ Metuximab is a murine mAb against CD147 (HAb18G), an extracellular matrix metalloproteinase inducer associated with hepatocarcinogenesis and HCC grading. ${ }^{30,33}$ Results from our systematic review suggest that Licartin had a beneficial effect on overall survival and recurrence-free survival of patients with HCC.

\section{Combined immunotherapy.}

Patients with HCC often have a compromised immune system due to liver cirrhosis, decreased level of lymphocytes, high levels of TGF- $\beta$, IL-4, IL-10, or regulatory T cells. Therefore, applying passive immunotherapy can improve anti-tumor immune responses induced by active immunotherapy. ${ }^{8,10,39}$ Three studies investigated combined immunotherapeutic approaches including ATVAC (autologous tumor lysate-pulsed dendritic cells plus activated $\mathrm{T}$ cell transfer) and autologous DC-CIK cell therapy in patients with HCC and only ATVAC improved overall survival of patients. Activated T cells administered in ATVAC can improve the induction of antitumor response by autologous tumor lysatepulsed DCs particularly in patients with HCC who have a compromised immune system. $8,11,34,41$

$a$-Galactosyl epitope is an antigen which is absent in normal human cells and therefore, natural antiGal antibody is abundant in serum. ${ }^{36}$ In the approach used by Qiu et al., DCs were pulsed with lysate tumor cells expressing $a$-Gal epitope which resulted in insitu binding of the patient's natural anti-Gal IgG and consequently DC opsonization and enhanced uptake of tumor antigens by DCs. ${ }^{36}$ Then these pulsed DCs were co-cultured with CIK cells to induce tumor-specific CIKs from naïve CIKs. ${ }^{36}$ In addition, in this study, newly differentiated CIKs from bone marrow stem cells were used instead of circulating $\mathrm{T}$ cells (which are compromised due to the overall immunocompromised state of patients). ${ }^{36}$ However, this approach did not result in any significant beneficial effect on survival in patients with HCC.

\section{Limitations.}

This meta-analysis had some limitations. First, there were considerable clinical heterogeneity in designs, interventions and populations (regarding stage and etiology of HCC and race of patients) of studies included in this systematic review. To address this issue, we collected details of each study and presented these details in a homogenous format in a table. In addition, we used random effects and sub-group meta-analysis based on different approaches of immunotherapy used in these studies. However, we did not use a 
subgroup meta-analysis based on study populations considering that few number of studies would be included in each sub-group.

Another source of heterogeneity was the designs and methods used in each study and consequently quality of studies. Therefore, we rated quality of studies using systematic methods of Cochrane collaboration and employed subgroup meta-analysis and randomeffects model.

Second, some outcomes in some studies were not reported properly so we could not include them in our meta-analysis. Therefore, we converted their results to the most interpretable outcome measures. For example, we have converted results of Kaplan-Meyers survival curves to HR or raw data of survival to risk ratio or median differences of survival time In addition, some clinical outcomes like recurrence-free survival were not reported in some studies. We tried to address this in quality rating of studies under selective reporting domain in Table 2. Third, the risk of bias was high in some studies and enough information was not reported to allow judgment of bias in particular domains in several articles. Fourth, number of included studies and sample sizes were small for some immunotherapeutic approaches like autologous DC vaccines studied by Ansary etal, Gao et al. and Nakamoto et al. which limited statistical power of detecting a significant difference between experimental groups and controls.

\section{Conclusions.}

Immunotherapy had extensive and varied use in the treatment of HCC. The systemic analysis presented herein illustrates the potential value of these approaches especially passive immunotherapies like CIK cell therapies, even though, no firm conclusions can be drawn due to the heterogeneity of the data available. Unlike, non-selective effects of conventional treatments, immunotherapy, theoretically, could selectively target and destroy malignant cells with minimal side effects. The advent of checkpoint inhibitors may help shed a better light on this modality of treatment and its potential value for HCC.

\section{Supplementary Material}

Refer to Web version on PubMed Central for supplementary material.

\section{References}

1. Jemal A, Bray F, Center MM, Ferlay J, Ward E, Forman D. Global cancer statistics. CA Cancer J. Clin 2011; 61: 69-90. [PubMed: 21296855]

2. Yao FY, Ferrell L, Bass NM et al. Liver transplantation for hepatocellular carcinoma: expansion of the tumor size limits does not adversely impact survival. Hepatology 2001; 33: 1394-403. [PubMed: 11391528]

3. El-Serag HB, Marrero JA, Rudolph L, Reddy KR. Diagnosis and treatment of hepatocellular carcinoma. Gastroenterology 2008; 134: 1752-63. [PubMed: 18471552]

4. Rougier P, Mitry E, Barbare JC, Taieb J. Hepatocellular carcinoma (HCC): an update. Semin. Oncol 2007; 34 (2 Suppl 1): S12-20. [PubMed: 17449346]

5. Parks AL, McWhirter RM, Evason K, Kelley RK. Cases of spontaneous tumor regression in hepatobiliary cancers: implications for immunotherapy? J. Gastrointest. Cancer 2015; 46: 161-5. [PubMed: 25662892] 
6. Wirth TC. Spontaneous and therapeutic immune responses in hepatocellular carcinoma: implications for current and future immunotherapies. Expert Rev. Gastroenterol. Hepatol 2014; 8: 101-10. [PubMed: 24410473]

7. Kawata A, Une Y, Hosokawa M et al. Adjuvant chemoimmunotherapy for hepatocellular carcinoma patients. Adriamycin, interleukin-2, and lymphokine-activated killer cells versus adriamycin alone. Am. J. Clin. Oncol 1995; 18: 257-62. [PubMed: 7747715]

8. Butterfield LH. Immunotherapeutic strategies for hepatocellular carcinoma. Gastroenterology 2004; 127: S232-1. [PubMed: 15508089]

9. Kirkwood JM, Butterfield LH, Tarhini AA, Zarour H, Kalinski P, Ferrone S. Immunotherapy of cancer in 2012. CA Cancer J. Clin 2012; 62: 309-35. [PubMed: 22576456]

10. Pardee AD, Butterfield LH. Immunotherapy of hepatocellular carcinoma: unique challenges and clinical opportunities. Oncoimmunology 2012; 1: 48-55. [PubMed: 22720211]

11. Butterfield LH. Recent advances in immunotherapy for hepatocellular cancer. Swiss Med. Wkly 2007; 137: 83-90. [PubMed: 17370144]

12. Kuang M, Peng BG, Lu MD et al. Phase II randomized trial of autologous formalin-fixed tumor vaccine for postsurgical recurrence of hepatocellular carcinoma. Clin. Cancer Res 2004; 10: 15749. [PubMed: 15014006]

13. Peng B, Liang L, Chen $\mathrm{Z}$ et al. Autologous tumor vaccine lowering postsurgical recurrent rate of hepatocellular carcinoma. Hepatogastroenterology 2006; 53: 409-14. [PubMed: 16795983]

14. Baogang P, Lijian L, Shuqin L et al. Fixed-tumor vaccine: a practical formulation with cytokinemicrospheres for protective and therapeutic antitumor immunity. Chin.-Ger J. Clin. Oncol 2003; 2: 196-202

15. Peng BG, Liang LJ, He Q et al. Tumor vaccine against recurrence of hepatocellular carcinoma. World J. Gastroenterol 2005; 11: 700-4. [PubMed: 15655825]

16. Gao J, Chen M, Ren H. Clinical effects of dendritic cells pulsed with autologous hepatoma cell lysates on the postoperative recurrence and metastasis of hepatocellular carcinoma. Chin. J. Hepatol 2005; 13: 432-5.

17. El Ansary M, Mogawer S, Elhamid SA et al. Immunotherapy by autologous dendritic cell vaccine in patients with advanced HCC. J. Cancer Res. Clin. Oncol 2013; 139: 39-8. [PubMed: 22886490]

18. Nakamoto Y, Mizukoshi E, Tsuji H et al. Combined therapy of transcatheter hepatic arterial embolization with intratumoral dendritic cell infusion for hepatocellular carcinoma: clinical safety. Clin. Exp. Immunol 2007; 147: 296-305. [PubMed: 17223971]

19. Lee JH, Lee JH, Lim YS et al. Adjuvant immunotherapy with autologous cytokine-induced killer cells for hepatocellular carcinoma. Gastroenterology 2015; 148: 1383-91 e6. [PubMed: 25747273]

20. Huang ZM, Li W, Li S et al. Cytokine-induced killer cells in combination with transcatheter arterial chemoembolization and radiofrequency ablation for hepatocellular carcinoma patients. J. Immunother 2013; 36: 287-93. [PubMed: 23719239]

21. Weng DS, Zhou J, Zhou QM et al. Minimally invasive treatment combined with cytokine-induced killer cells therapy lower the shortterm recurrence rates of hepatocellular carcinomas. J. Immunother 2008; 31: 63-71. [PubMed: 18157013]

22. Zhou QM, Wu PH, Zhao M et al. Short-term curative efficacy of cytokine-induced killer cells combined micro-invasive treatments on hepatocellular carcinoma. Chin. J. Cancer 2006; 25: 1414 8.

23. Zhao M, Wu PH, Zeng YX et al. Cytokine-induced killer cell fusion to lower recurrence of hepatocellular carcinoma after transcatheter arterial chemoembolization sequentially combined with radiofrequency ablation: a randomized trial. Zhonghua Yi Xue Za Zhi 2006; 86: 1823-8. [PubMed: 17054858]

24. Pan CC, Huang ZL, Li W et al. Serum alpha-fetoprotein measurement in predicting clinical outcome related to autologous cytokine-induced killer cells in patients with hepatocellular carcinoma undergone minimally invasive therapy. Chin. J. Cancer 2010; 29: 596-602. [PubMed: 20507732]

25. Hao MZ, Lin HL, Chen Q, Ye YB, Chen QZ, Chen MS. Efficacy of transcatheter arterial chemoembolization combined with cytokine-induced killer cell therapy on hepatocellular carcinoma: a comparative study. Chin. J. Cancer 2010; 29: 172-7. [PubMed: 20109346] 
26. Yu X, Zhao H, Liu L et al. A randomized phase II study of autologous cytokine-induced killer cells in treatment of hepatocellular carcinoma. J. Clin. Immunol 2014; 34: 194-203. [PubMed: 24337625]

27. Hui D, Qiang L, Jian W, Ti Z, Da-Lu K. A randomized, controlled trial of postoperative adjuvant cytokine-induced killer cells immunotherapy after radical resection of hepatocellular carcinoma. Dig. Liver Dis 2009; 41: 36-1. [PubMed: 18818130]

28. Takayama T, Sekine T, Makuuchi M et al. Adoptive immunotherapy to lower postsurgical recurrence rates of hepatocellular carcinoma: a randomised trial. Lancet 2000; 356: 802-7. [PubMed: 11022927]

29. Uchino J, Une Y, Kawata A, Wakisaka Y, Hosokawa M. Postoperative chemoimmunotherapy for the treatment of liver cancer. Semin. Surg. Oncol 1993; 9: 332-6. [PubMed: 8210915]

30. Bian H, Zheng JS, Nan G et al. Randomized trial of $\left[{ }^{131} \mathrm{I}\right]$ metuximab in treatment of hepatocellular carcinoma after percutaneous radiofrequency ablation. J. Natl. Cancer Inst 2014; 106.

31. Zeng ZC, Tang ZY, Liu KD, Lu JZ, Xie H, Yao Z. Improved long-term survival for unresectable hepatocellular carcinoma (HCC) with a combination of surgery and intrahepatic arterial infusion of 131I-anti-HCC mAb. Phase I/II clinical trials. J. Cancer Res. Clin. Oncol 1998; 124: 275-80. [PubMed: 9645459]

32. Order S, Pajak T, Leib S et al. A randomized prospective trial comparing full dose chemotherapy to ${ }^{131}$ I antiferritin: An RTOG study. Int. J. Radiat. Oncol. Biol. Phys 1991; 20: 953-63. [PubMed: 1850722]

33. Xu J, Shen ZY, Chen XG et al. A randomized controlled trial of Licartin for preventing hepatoma recurrence after liver transplantation. Hepatology 2007; 45: 269-76. [PubMed: 17256759]

34. Shimizu K, Kotera Y, Aruga A et al. Postoperative dendritic cell vaccine plus activated T-cell transfer improves the survival of patients with invasive hepatocellular carcinoma. Hum. Vaccin 2014; 10: 970-6.

35. Liu L, CXxDNQQQZ Zhong J. Transarterial chemoembolization combined with autologous DC CIK cells for the treatment of hepatocellular carcinoma of BCLC stage: a randomized controlled study. J Intervent Radiol. 2015; 24: 434-8.

36. Qiu Y, Xu MB, Yun MM et al. Hepatocellular carcinoma-specific immunotherapy with synthesized alpha1,3- galactosyl epitope-pulsed dendritic cells and cytokine-induced killer cells. World J. Gastroenterol 2011; 17: 5260-6. [PubMed: 22219594]

37. Kalinski P, Edington H, Zeh HJ et al. Dendritic cells in cancer immunotherapy: vaccines or autologous transplants? Immunol. Res 2011; 50: 235-47. [PubMed: 21717071]

38. Zhong JH, Ma L, Wu LC et al. Adoptive immunotherapy for postoperative hepatocellular carcinoma: a systematic review. Int. J. Clin. Pract 2012; 66: 21-7. [PubMed: 22171902]

39. Willimsky G, Protzer U, Knolle P, Heikenwalder M. Adoptive T-cell therapy to treat liver cancer: is the liver microenvironment key? Oncotarget 2013; 4: 1117-8. [PubMed: 23907631]

40. Galluzzi L, Vacchelli E, Eggermont A et al. Trial Watch: Adoptive cell transfer immunotherapy. Oncoimmunology 2012; 1: 306-15. [PubMed: 22737606]

41. West EJ, Scott KJ, Jennings VA, Melcher AA. Immune activation by combination human lymphokine-activated killer and dendritic cell therapy. Br. J. Cancer 2011; 105: 787-95. [PubMed: 21847125]

42. Zang YW, Gu XD, Xiang JB, Chen ZY. Clinical application of adoptive T cell therapy in solid tumors. Med. Sci. Monit 2014; 20: 953-9. [PubMed: 24912947] 

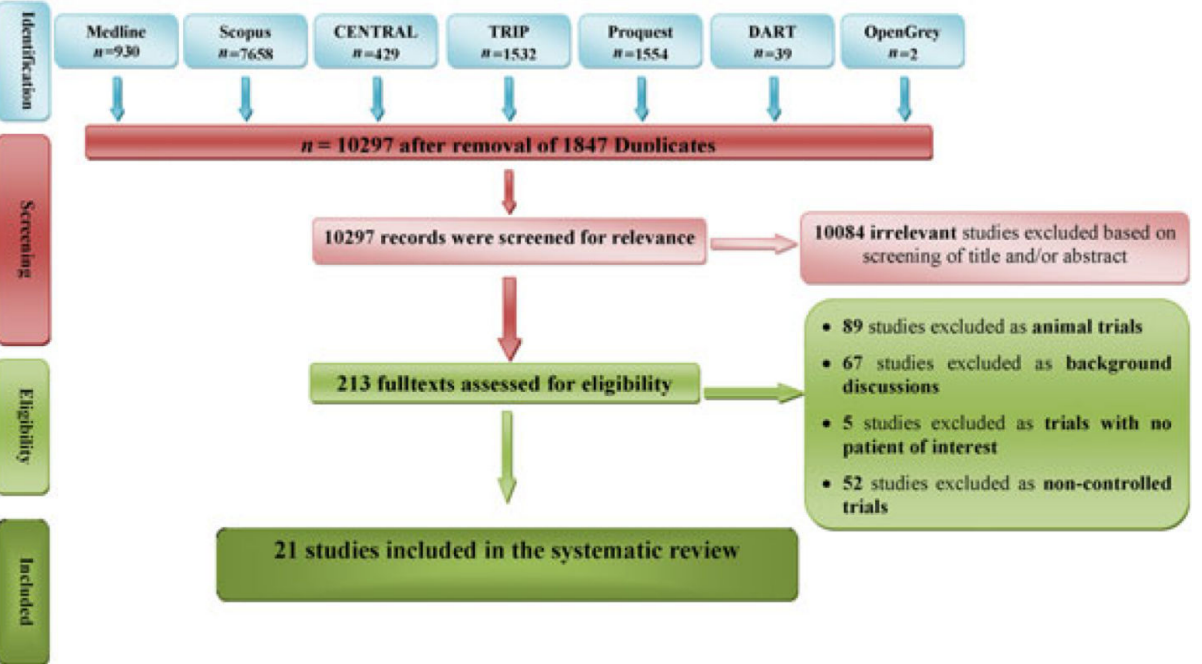

21 studies included in the systematic review

Figure 1.

Study flow diagram. [Color figure can be viewed at wileyonlinelibrary.com] 


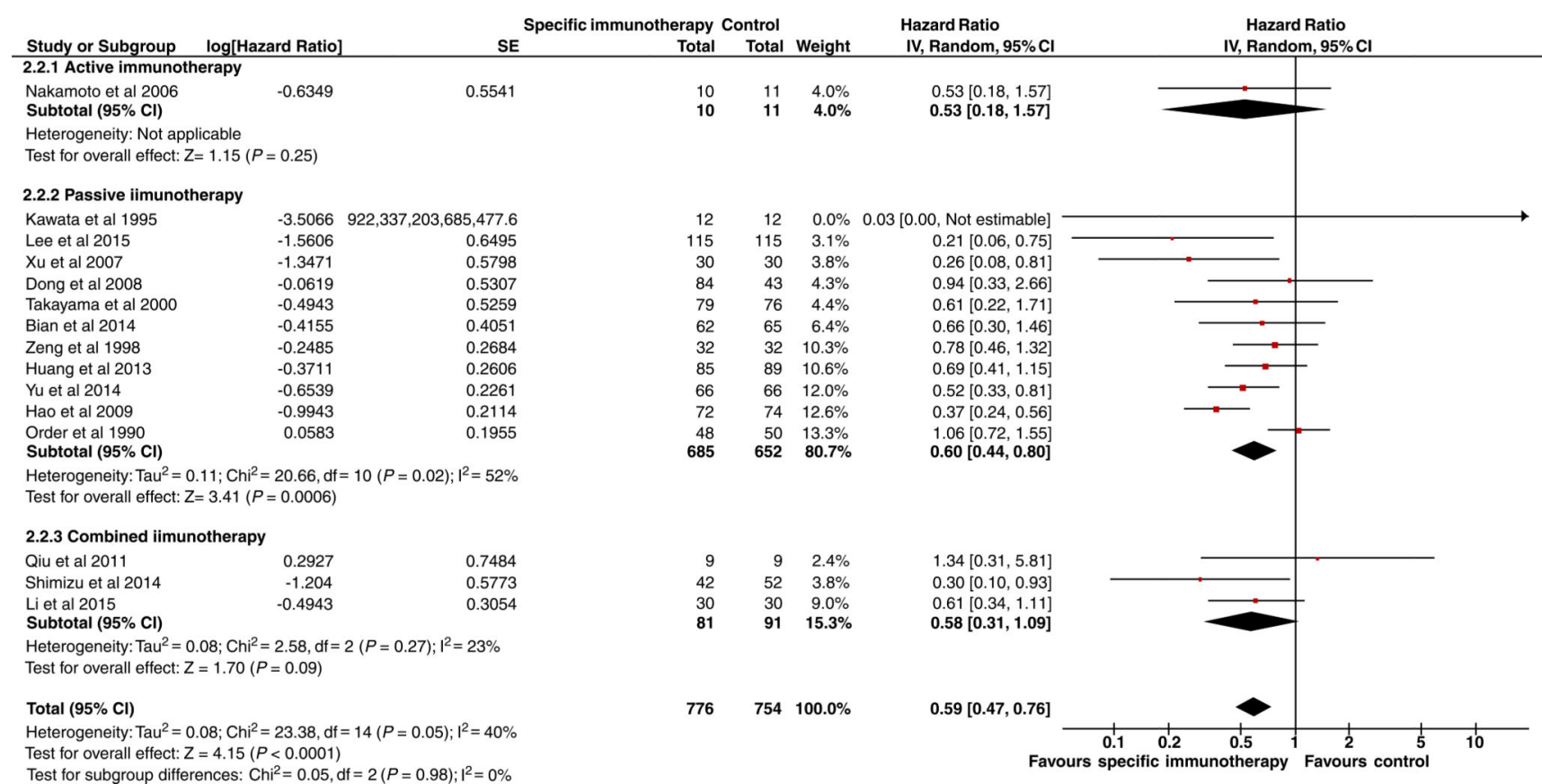

Figure 2.

Comparison of overall survival between patients in specific immunotherapy group and control group. [Color figure can be viewed at wileyonlinelibrary.com] 


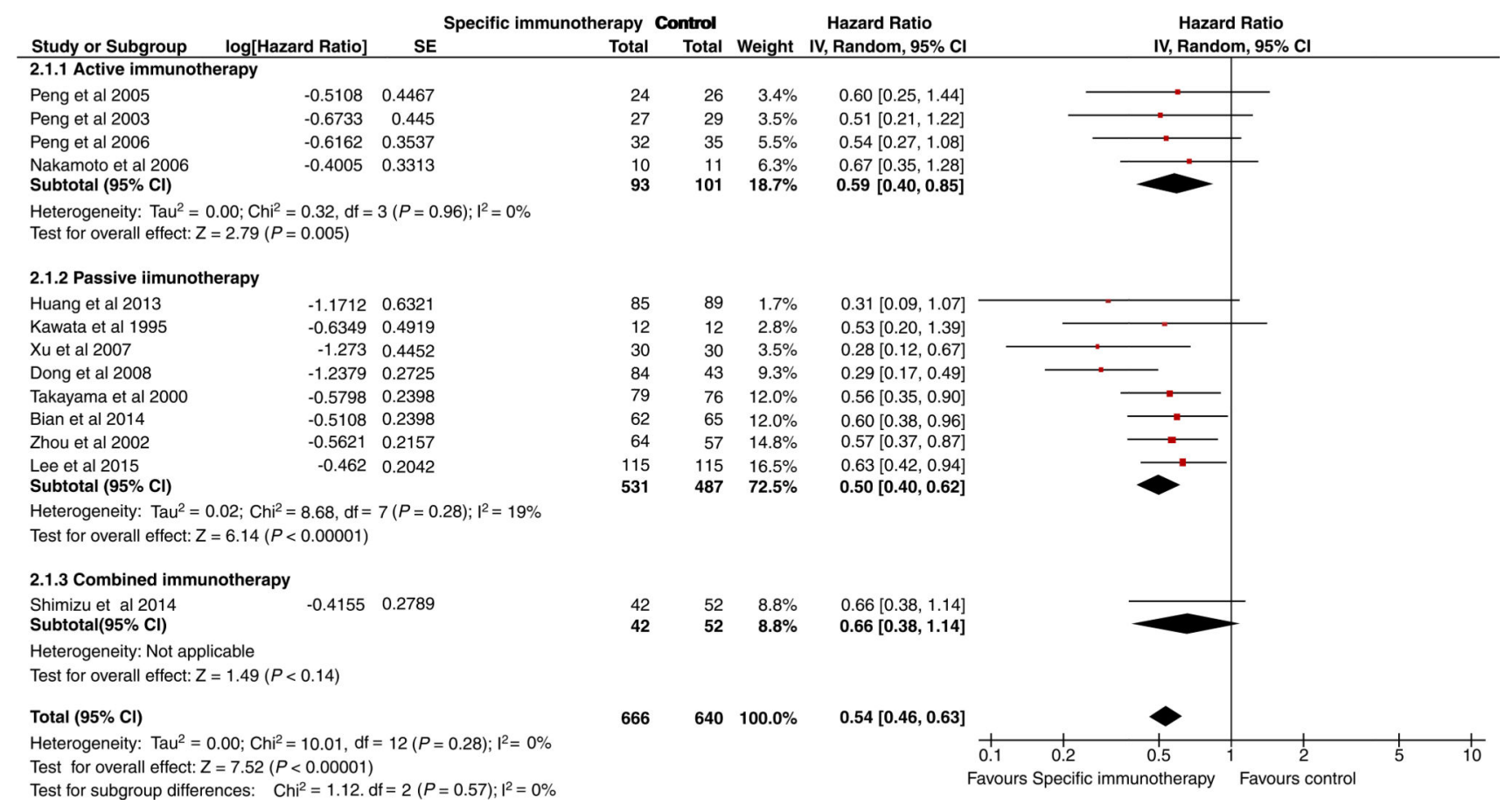

Figure 3.

Comparison of recurrence-free survival between patients in specific immunotherapy group and control group. [Color figure can be viewed at wileyonlinelibrary.com] 


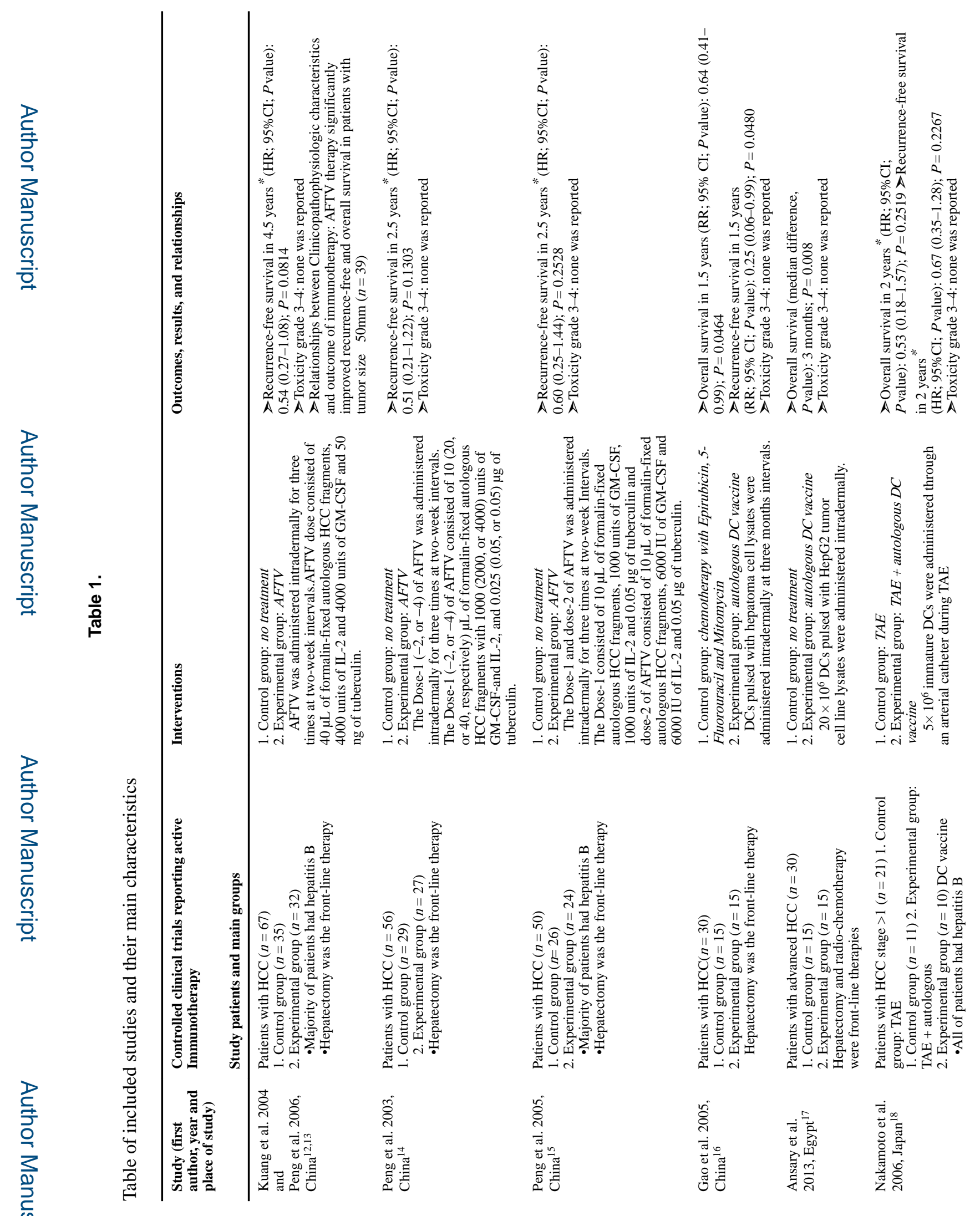

J Gastroenterol Hepatol. Author manuscript; available in PMC 2019 February 15. 


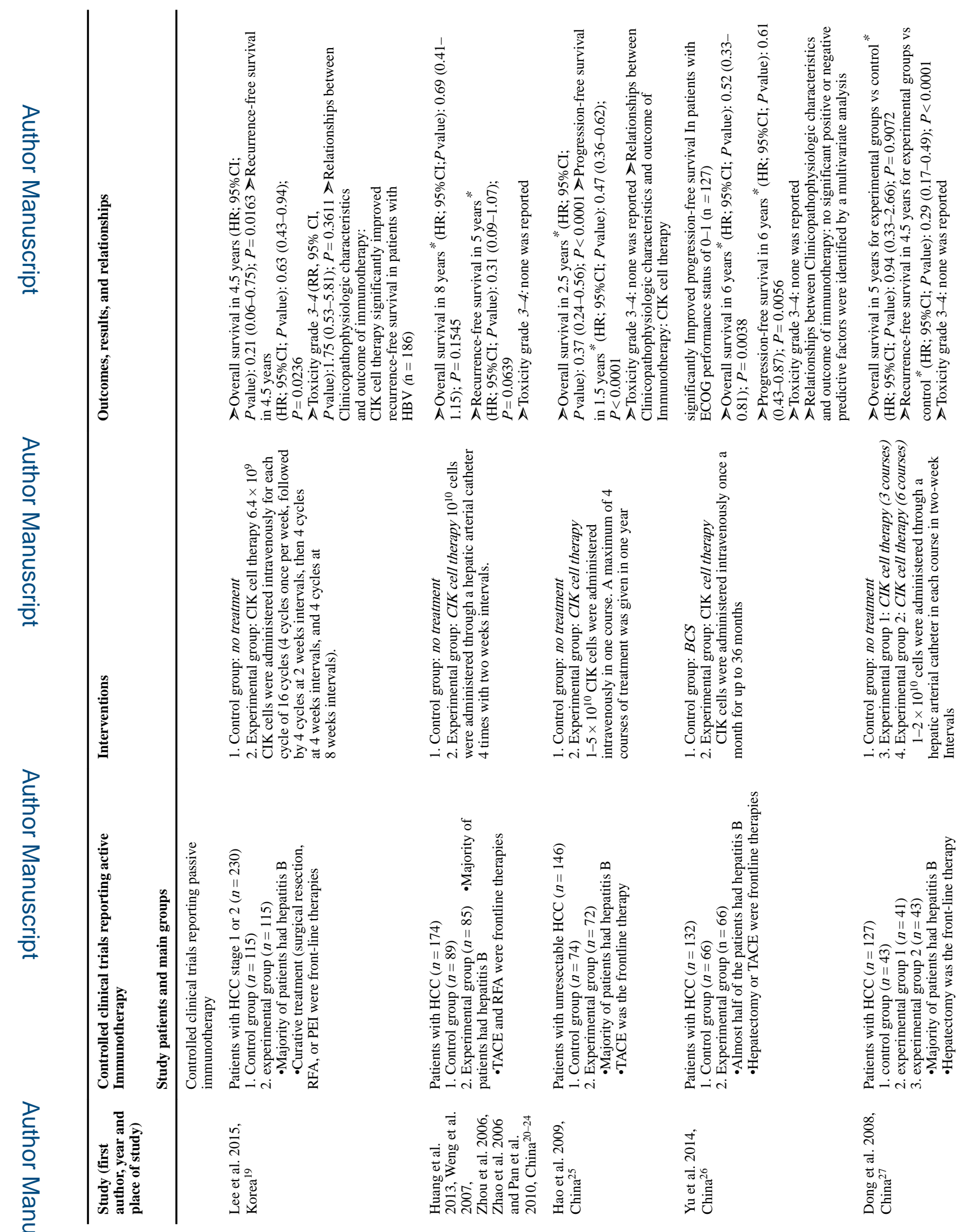




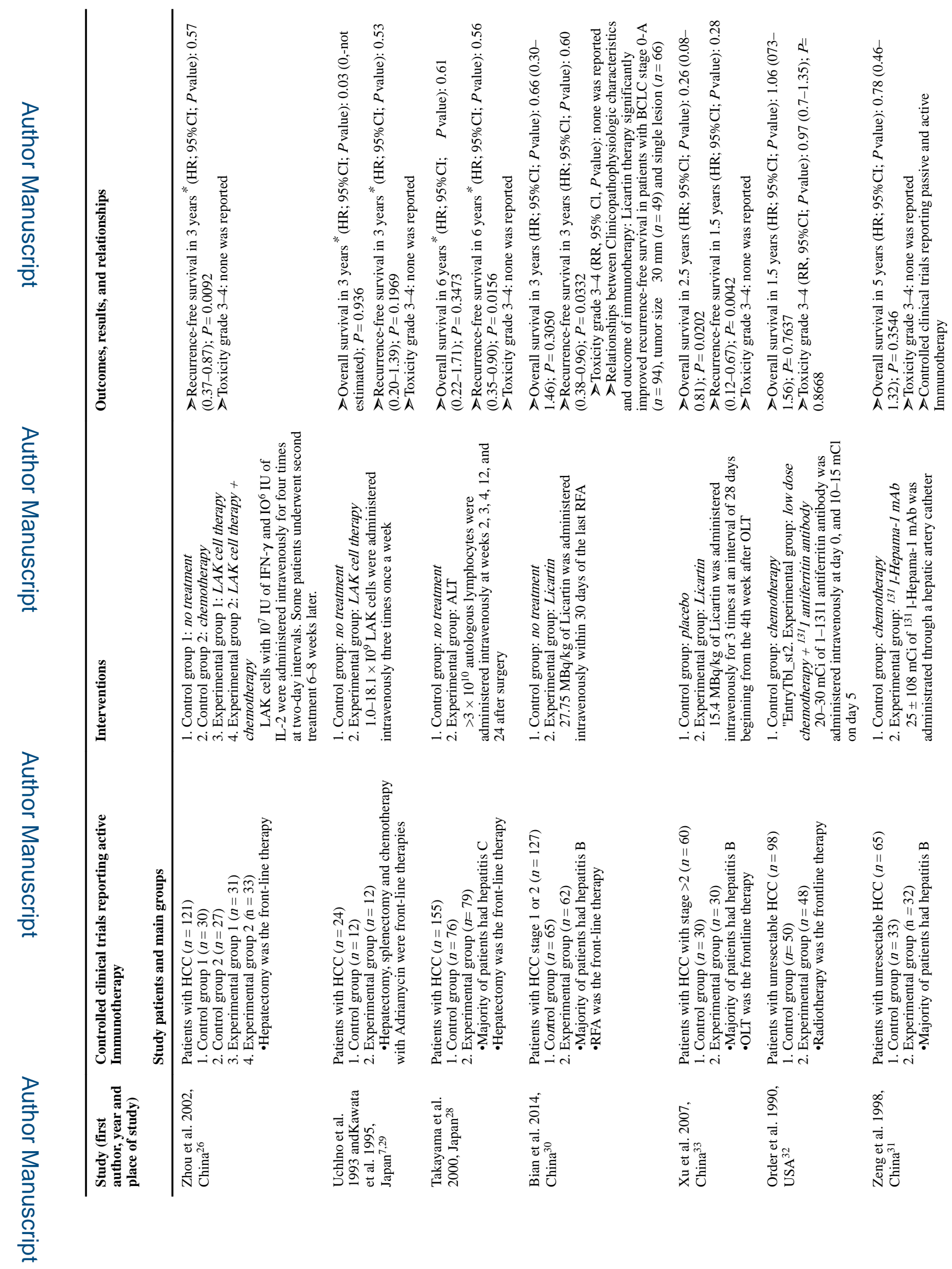

J Gastroenterol Hepatol. Author manuscript; available in PMC 2019 February 15. 
Noveiry et al.

Page 18

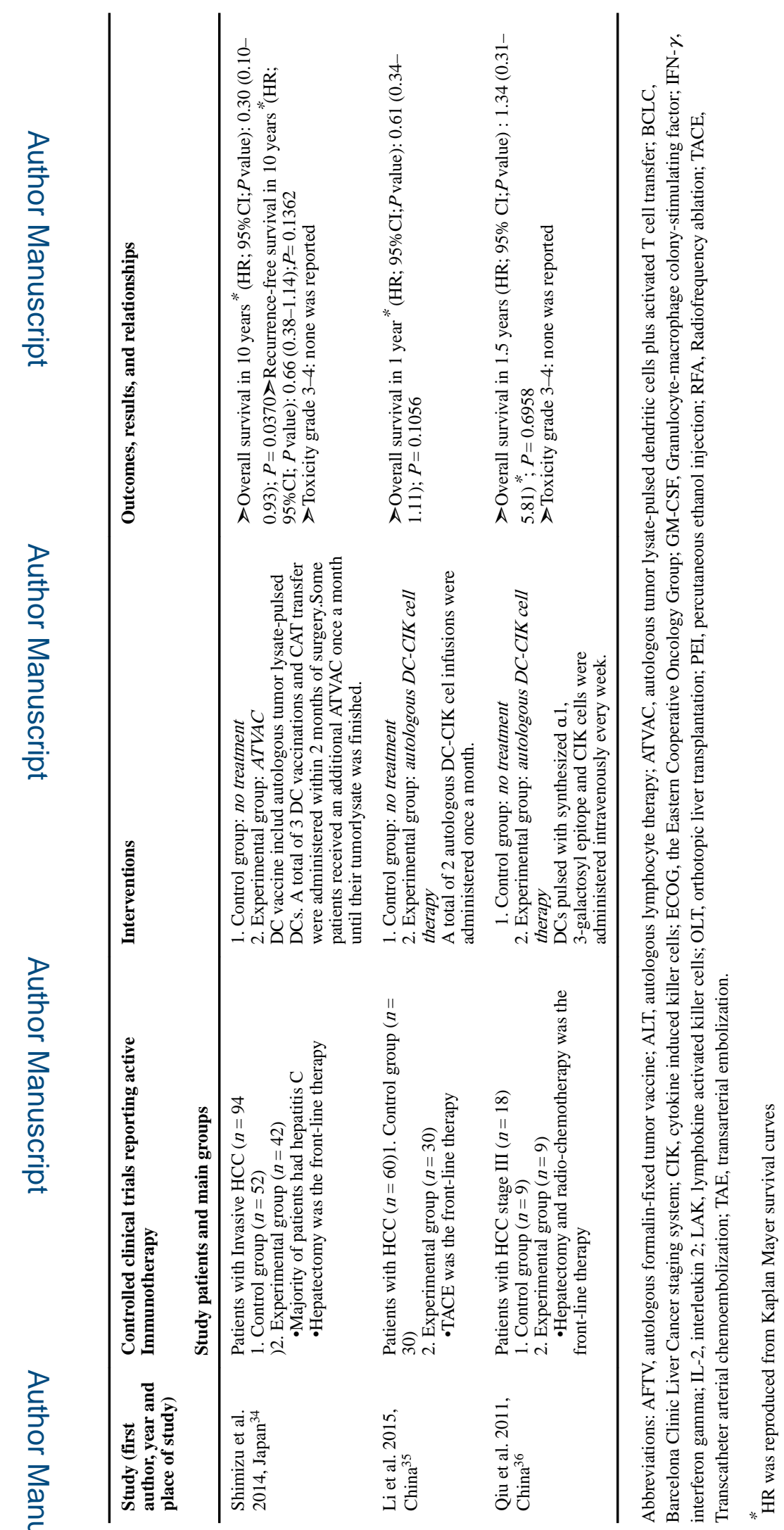

J Gastroenterol Hepatol. Author manuscript; available in PMC 2019 February 15. 
Table 2

Risk of bias in included studies

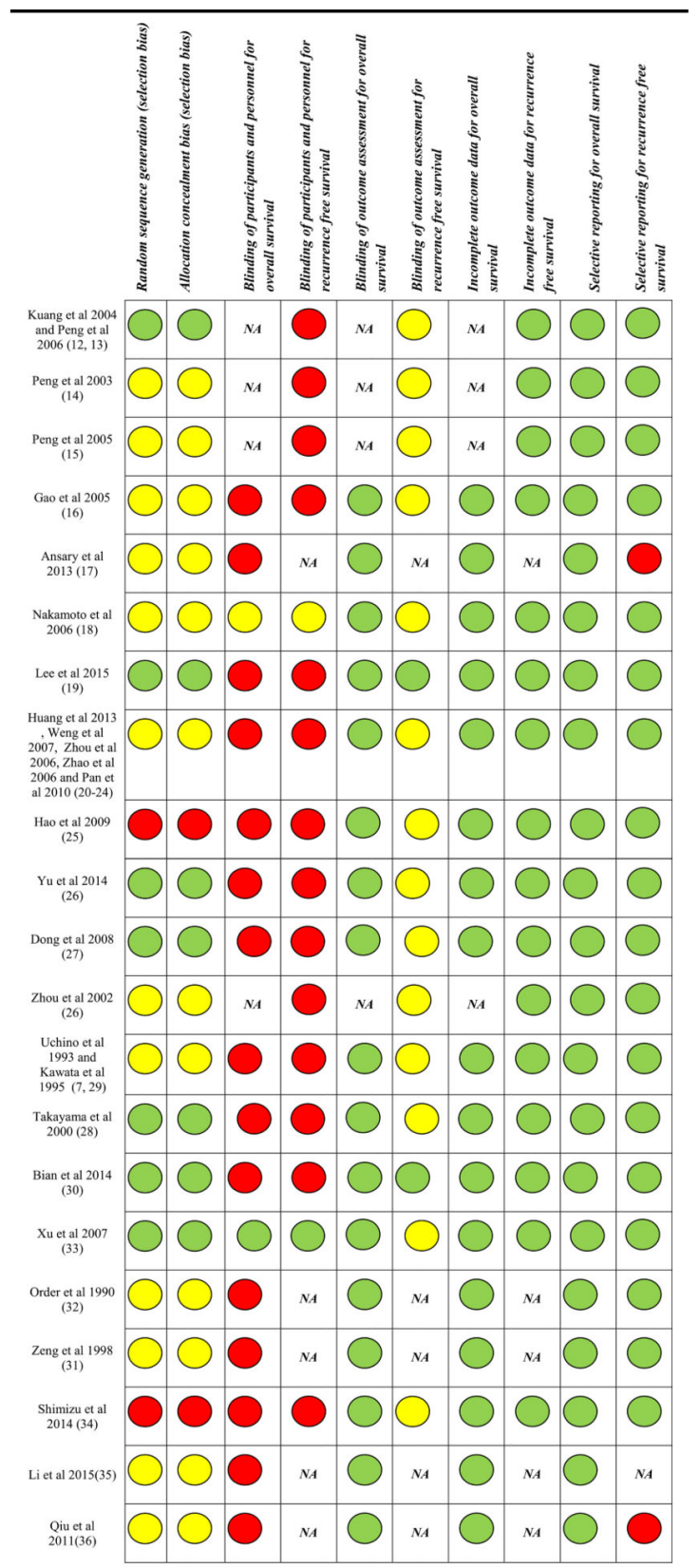

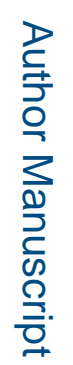

Low risk of bias

Unclear risk of bias

High risk of bias

J Gastroenterol Hepatol. Author manuscript; available in PMC 2019 February 15. 